\title{
The historical roots of carotid artery stenting literature: an analysis of cited references
}

\author{
Andy Wai Kan Yeung*
}

Carotid artery stenting (CAS) is a popular treatment option for carotid artery stenosis. Numerous studies have suggested that the work of Kerber et al. in 1980 was an important cornerstone for $C A S$. The primary objectives of the present study are to identify the historical roots of CAS and show that carotid endarterectomy (CEA) is highly relevant to CAS. For this, 69,570 references cited by 3380 articles were analysed. There were nine reference publication year spectroscopy peaks ranging from 1980 to 2011, and the publication by Kerber et al. was identified from the first peak. Nine key references were identified, all of which reported treatment outcomes or procedures of CAS and/or CEA.

Keywords: Carotid artery stenosis, citation analysis, cited references, endarterectomy, historical roots.

It has been reported that carotid artery stenting (CAS) was performed with a $98.9 \%$ success rate, with restenosis rates lower than $3 \%$ at $1-3$ years post-operatively ${ }^{1}$. With these encouraging numbers, it would be worthwhile to evaluate the scientific literature on this topic. Multiple sources $^{2,3}$ have pointed to the groundbreaking article by Kerber $e t a l .{ }^{4}$ that paved the way for CAS, as it described a case of successful insertion of a catheter to dilate a stenosed carotid artery in a patient. It should be noted that other research teams, such as one led by Mathias also performed balloon angioplasty during the same period ${ }^{3}$. CAS is often compared with carotid endarterectomy (CEA), which is another treatment option for carotid artery stenosis ${ }^{5,6}$. A recently published bibliometric study provided useful information on the CAS literature ${ }^{7}$. By identifying the 100 most cited publications, we now know that: (i) the United States has been the major contributor to these studies; (ii) Stroke was the lead productive journal and (iii) studies published in journals with higher impact factor were often cited more frequently ${ }^{7}$. The present study aimed to complement the study of Cheng et al. ${ }^{7}$ by evaluating the CAS literature from a different perspective. Instead of analysing the most cited publications, the cited references of CAS publications were analysed. In particular, the present study was designed to address three research questions:

(i) Can the analysis of cited references show that CEA is highly relevant to CAS?

(ii) How did key references compare CAS with CEA?

Andy Wai Kan Yeung is in the Oral and Maxillofacial Radiology, Applied Oral Sciences and Community Dental Care, Faculty of Dentistry, The University of Hong Kong, Hong Kong, China.

*e-mail: ndyeung@hku.hk (iii) Can the analysis of cited references identify seminal works that are the historical roots of CAS?

Most of the conventional bibliometric analysis on citation data of publications resulted from a search with a pre-defined string ${ }^{7}$, which, in this case, should intuitively be 'CAS' or 'carotid artery stenting'. However, the seminal works frequently cited by CAS publications might not include these phrases, because either they might have been published before such phrases became well-established, or they were not directly addressing CAS. To address the above-mentioned research questions, a cited reference analysis was performed using CRExplorer ${ }^{8}$. This software can plot a 'reference publication year spectroscopy' (RPYS), which resembles a waveform along a horizontal timeline, and the waveform can demonstrate in which years the more frequently cited references (compared to preceding and succeeding years) were published $^{9-12}$.

\section{Material and methods}

Data were extracted from the Web of Science Core Collection online database on 27 February 2019. A search was performed using the following strategy: TOPIC = ('carotid artery stent*'). This search string closely followed that of Cheng et al. ${ }^{7}$, but did not include the abbreviation CAS because this has multiple meanings, for example, Cas9 - an enzyme associated with the CRISPRCas 9 genome-editing tool. The search did not place additional restrictions, such as journal category, publication year or language. The search yielded 3380 publications, between 1996 and 2019, which collectively had 69,570 cited references. 


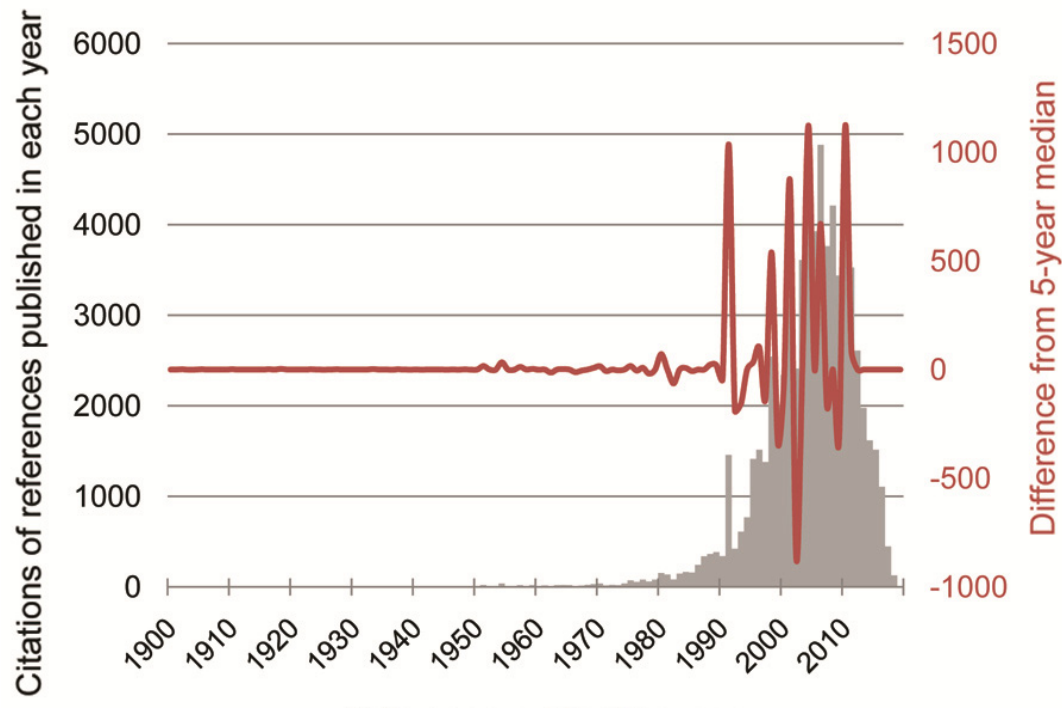

Reference publication year

Figure 1. Results from reference publication year spectroscopy. The reference lists of the 3380 carotid artery stenting publications were analysed using CRExplorer. References were sorted by publication year ( $x$-axis), and the citations received by each reference published in the same year were added (grey, $y$-axis on the left). The waveform showed the fluctuations in the difference in annual citation count from its 5-yr median (red, $y$-axis on the right).

Table 1. Key references identified by reference publication year spectroscopy

\begin{tabular}{|c|c|c|c|c|c|c|}
\hline Year & Reference & $\begin{array}{l}\text { No. of times } \\
\text { cited by } 3380 \\
\text { publications }\end{array}$ & $\begin{array}{l}\text { No. of times } \\
\text { cited by all pub- } \\
\text { lications indexed } \\
\text { in the Web of } \\
\text { Science (WoS) }\end{array}$ & $\begin{array}{l}\text { Percentage } \\
\text { contribu- } \\
\text { tion to the } \\
\text { peak }\end{array}$ & $\begin{array}{l}\text { No. of times in the top } \\
10 \% \text { cited reference of } \\
\text { a year, according to the } \\
\text { dataset }\end{array}$ & $\begin{array}{c}\text { Relevance to } \\
\text { carotid artery stenting (CAS) }\end{array}$ \\
\hline 1980 & 4 & 27 & 139 & 17.5 & 13 & $\begin{array}{l}\text { Reported catheter dilatation during } \\
\text { carotid endarterectomy (CEA) }\end{array}$ \\
\hline 1996 & 24 & 141 & 452 & 9.3 & 23 & Reported clinical experiences with CAS* \\
\hline 1998 & 25 & 294 & 2109 & 11.6 & 20 & $\begin{array}{l}\text { Investigated the beneficial effects of } \\
\text { CEA }\end{array}$ \\
\hline 2001 & 16 & 311 & 820 & 9.5 & 19 & $\begin{array}{l}\text { Compared treatment outcomes of CAS } \\
\text { and CEA* }\end{array}$ \\
\hline 2004 & 18 & 851 & 1748 & 16.9 & 16 & $\begin{array}{l}\text { Compared treatment outcomes of CAS } \\
\text { and CEA* }\end{array}$ \\
\hline 2006 & 17 & 458 & 1005 & 9.4 & 13 & $\begin{array}{l}\text { Compared treatment outcomes of CAS } \\
\text { and CEA* }\end{array}$ \\
\hline 2010 & 6 & 683 & 1447 & 14.7 & 10 & $\begin{array}{l}\text { Compared treatment outcomes of CAS } \\
\text { and CEA* }\end{array}$ \\
\hline 2011 & 26 & 92 & 121 & 2.6 & 7 & $\begin{array}{l}\text { Investigated cerebral protection } \\
\text { procedures during CAS }\end{array}$ \\
\hline
\end{tabular}

*Also identified by Cheng et al. ${ }^{7}$.

The full record and cited references of the 3380 CAS publications were imported into the CRExplorer software, which is capable of identifying publications, within a predefined body of the literature, that are most frequently referenced ${ }^{10-14}$. The RPYS plot is composed of two parts: a bar chart that shows the raw frequency of cited references published in each publication year, and a waveform that shows positive and negative peaks which indicate publication years when the citation count largely deviated from its 5-yr median. For instance, references published in 2008-2012 were cited 4211, 3437, 4648, 3526 and 2609 times respectively. The 5 -yr median was 3526 . Hence references published in 2010 , being cited 4648 times, were cited 1122 times more than its 5-yr median, and formed a positive peak with a magnitude of 1122 . Positive peaks with a magnitude of at least 50 were examined. To focus on the more important references, only those with $>10 \%$ contributions to the peak were 
identified $^{15}$. If in a peak there was no reference with $>10 \%$ contribution, the one with the highest contribution was identified. Moreover, for the important references identified, the N_TOP10 indicator was examined, which recorded the number of years when a reference was in the top $10 \%$ cited according to the analysed dataset.

\section{Results}

There were nine peaks identified in the years 1980, 1991, 1996, 1998, 2001, 2004, 2006 and 2010-2011 (Figure 1). The largest peaks were in the 1990 s-2010s. Table 1 provides details of the nine key references that satisfied our pre-defined criteria. Four of them were not identified by the conventional citation analysis reported by Cheng et $a l .^{7}$. They have been among the top $10 \%$ cited references for 7-23 years. Using the RPYS method, the publication of Kerber et al. ${ }^{4}$ was identified from the first peak. It was responsible for $17.5 \%$ of citations made to references published in that year. A total of nine references were identified, four of which compared the treatment outcomes of CAS and CEA, two investigated the beneficial effects of CEA, one reported the clinical experiences with $\mathrm{CAS}$, one investigated the cerebral protection procedures during CAS, and one reported catheter dilatation during CEA. Many of these references were published in the New England Journal of Medicine with an impact factor of 70.670, suggesting that they had substantial scientific merit and high impact.

\section{Discussion}

Cheng et $a l .^{7}$ analysed the 100 most cited CAS publications and found that they were published between 1992 and 2016, with a large proportion during the 2000s ( $n=68)$, in the journal Stroke $(n=21)$, and compared the efficacy and safety with CEA $(n=28)$. In the present study, nine key references cited by CAS publications were published between 1980 and 2011. Majority of them $(n=5)$ were published in the New England Journal of Medicine, while none was published in Stroke. Four of them compared CAS with CEA. By considering both bibliometric studies, one can notice that the comparison of CAS and CEA has been a hot topic for research in neurosurgery. Moreover, in terms of all-time citations, CAS publications in Stroke were outstanding. On the other hand, many of the key references cited by CAS publications were published in the New England Journal of Medicine.

By analysing cited references with RPYS, we were able to demonstrate that CEA is highly relevant to CAS. Moreover, the four key references that compared CAS with CEA reported mixed results regarding which one provided a more superior outcome $e^{6,16-18}$. The present study did not aim to compare the treatment outcomes of
CAS and CEA, as readers may refer to multiple metaanalyses published regarding this issue ${ }^{19-22}$. Instead, the focus here was to highlight that the citations to these publications were not biased to favour either CAS or CEA. In addition, the analysis was also able to identify the publication of Kerber et al. ${ }^{4}$ from the first peak, showing that it was an important reference for CAS publications. RPYS also identified four references not reported by Cheng et ll $^{7}$, demonstrating that this method could overcome the limitations of conventional citation analysis, such as the problem of not being identified by definitive search words, or all-time most cited.

The present analysis had some limitations. First, some potentially important but rarely cited references might be unnoticed. Second, publications not indexed in the Web of Science have not been analysed, as well as their references.

\section{Conclusion}

This study provides a novel perspective on the CAS literature. In particular, that many of the key references were published in the New England Journal of Medicine, and also related to comparisons between CAS and CEA. Key references could be traced back to as early as the 1980 s.

Conflict of interest. The author declares no potential conflict of interest.

1. Wholey, M. H., Al-Mubarek, N. and Wholey, M. H., Updated review of the global carotid artery stent registry. Catheter Cardiovasc. Interv., 2003, 60, 259-266.

2. Saw, J., Carotid artery stenting for stroke prevention. Can. J. Cardiol., 2014, 30, 22-34.

3. Roffi, M. and Mathias, K., History of carotid artery stenting. $J$. Cardiovasc. Surg., 2013, 54, 1-10.

4. Kerber, C. W., Cromwell, L. D. and Loehden, O. L., Catheter dilatation of proximal carotid stenosis during distal bifurcation endarterectomy. Am. J. Neuroradiol., 1980, 1, 348-349.

5. Alberts, M. J., Results of a multicenter prospective randomized trial of carotid artery stenting vs carotid endarterectomy. Stroke, 2001, 32, 325.

6. Brott, T. G. et al., Stenting versus endarterectomy for treatment of carotid-artery stenosis. N. Engl. J. Med., 2010, 363, 11-23.

7. Cheng, W., Kang, Q. and Xiao, Q., Bibliometric analysis of the top 100 influential studies on carotid artery stenting. World Neurosurg., 2019, 122, e1321-e1331.

8. Thor, A., Marx, W., Leydesdorff, L. and Bornmann, L., Introducing CitedReferencesExplorer (CRExplorer): a program for reference publication year spectroscopy with cited references standardization. J. Informetr., 2016, 10, 503-515.

9. Marx, W. and Bornmann, L., Tracing the origin of a scientific legend by reference publication year spectroscopy (RPYS): the legend of the Darwin finches. Scientometrics, 2014, 99, 839-844.

10. Marx, W., Bornmann, L., Barth, A. and Leydesdorff, L., Detecting the historical roots of research fields by reference publication year spectroscopy (RPYS). J. Assoc. Inf. Sci. Technol., 2014, 65, 751764. 
11. Wray, K. B. and Bornmann, L., Philosophy of science viewed through the lense of 'Referenced Publication Years Spectroscopy' (RPYS). Scientometrics, 2015, 102, 1987-1996.

12. Yeung, A. W. K., Wong, N. S. M. and Leung, Y. Y., Are coronectomy studies being cited? A bibliometric study. J. Invest. Clin. Dent., 2019, 10, e12366.

13. Yeung, A. W. K. and Wong, N. S. M., The historical roots of visual analogue scale in psychology as revealed by reference publication year spectroscopy. Front. Hum. Neurosci., 2019, 13, 86.

14. Yeung, A. W. K., Identification of seminal works that built the foundation for functional magnetic resonance imaging studies of taste and food. Curr. Sci., 2017, 113, 1225-1227.

15. Bornmann, L., Haunschild, R. and Leydesdorff, L., Reference publication year spectroscopy (RPYS) of Eugene Garfield's publications. Scientometrics, 2018, 114, 439-448.

16. Brown, M., Rogers, J. and Bland, J., Endovascular versus surgical treatment in patients with carotid stenosis in the Carotid and Vertebral Artery Transluminal Angioplasty Study (CAVATAS): a randomised trial. Lancet, 2001, 357, 1729-1737.

17. Mas, J.-L. et al., Endarterectomy versus stenting in patients with symptomatic severe carotid stenosis. N. Engl. J. Med., 2006, 355, 1660-1671.

18. Yadav, J. S. et al., Protected carotid-artery stenting versus endarterectomy in high-risk patients. N. Engl. J. Med., 2004, 351, 1493-1501.

19. Economopoulos, K. P., Sergentanis, T. N., Tsivgoulis, G., Mariolis, A. D. and Stefanadis, C., Carotid artery stenting versus carotid endarterectomy: a comprehensive meta-analysis of shortterm and long-term outcomes. Stroke, 2011, 42, 687-692.

20. Luebke, T., Aleksic, M. and Brunkwall, J., Meta-analysis of randomized trials comparing carotid endarterectomy and endovascular treatment. Eur. J. Vasc. Endovasc. Surg., 2007, 34, 470-479.

21. Murad, M. H., Flynn, D. N., Elamin, M. B., Guyatt, G. H., Hobson II, R. W., Erwin, P. J. and Montori, V. M., Endarterectomy vs stenting for carotid artery stenosis: a systematic review and metaanalysis. J. Vasc. Surg., 2008, 48, 487-493.

22. Murad, M. H., Shahrour, A., Shah, N. D., Montori, V. M. and Ricotta, J. J., A systematic review and meta-analysis of randomized trials of carotid endarterectomy vs stenting. J. Vasc. Surg., 2011, 53, 792-797.

23. North American Symptomatic Carotid Endarterectomy Trial Collaborators. Beneficial effect of carotid endarterectomy in symptomatic patients with high-grade carotid stenosis. New Eng. J. Med., 1991, 325(7), 445-453.

24. Diethrich, E. B., Ndiaye, M. and Reid, D. B., Stenting in the carotid artery: initial experience in 110 patients. J. Endovasc. Ther., 1996, 3(1), 42-62.

25. Barnett, H. J., Taylor, D. W., Eliasziw, M., Fox, A. J., Ferguson, G. G., Haynes, R. B. and Thorpe, K. E., Benefit of carotid endarterectomy in patients with symptomatic moderate or severe stenosis. New Eng. J. Med., 1998, 339(20), 1415-1425.

26. Montorsi, P., Caputi, L., Galli, S., Ciceri, E., Ballerini, G., Agrifoglio, M. and Loaldi, A., Microembolization during carotid artery stenting in patients with high-risk, lipid-rich plaque: a randomized trial of proximal versus distal cerebral protection. J. Am. College Cardiol., 2011, 58(16), 1656-1663.

Received 8 April 2019; revised accepted 10 June 2020

doi: $10.18520 / \mathrm{cs} / \mathrm{v} 119 / \mathrm{i} 3 / 447-450$ 